\title{
The Clinical Efficacy of Cefoperazone-sulbactam Versus Piperacillin-tazobactam in the Treatment of Severe Community-acquired Pneumonia
}

Chia-Hung Chen ( $\square$ hsnu758@gmail.com )

China Medical University Hospital

Chih-Cheng Lai

Kaohsiung Veterans General Hospital

Wei-Chih Chen

Taipei Veterans General Hospital

Li-Kuo Kuo

Mackay Memorial Hospital

Yao-Tung Wang

Chung Shan Medical University Hospital

Pin-Kuei Fu

Taichung Veterans General Hospital

Shih-Chi Ku

National Taiwan University Hospital

Wen-Feng Fang

Kaohsiung Chang Gung Memorial Hospital

Chin-Ming Chen

Chi Mei Medical Center

Chih-Yen Tu

China Medical University Hospital

Wen-Chien Cheng

China Medical University Hospital

\section{Research Article}

Keywords: cefoperazone-sulbactam, piperacillin-tazobactam, severe community-acquired pneumonia

Posted Date: January 17th, 2022

DOI: https://doi.org/10.21203/rs.3.rs-1077299/v1 
License: (c) (i) This work is licensed under a Creative Commons Attribution 4.0 International License. Read Full License 


\section{Abstract}

To compare the clinical efficacy of cefoperazone-sulbactam with piperacillin-tazobactam in the treatment of severe community-acquired pneumonia (SCAP). The retrospective study was conducted from March 1, 2018 to May 30, 2019. Clinical outcomes were compared for patients who received either cefoperazonesulbactam or piperacillin-tazobactam in the treatment of SCAP.

A total of 815 SCAP patients were enrolled. Among them, 343 received cefoperazone-sulbactam, and 472 received piperacillin-tazobactam. Patients who received cefoperazone-sulbactam presented with higher Charlson Comorbidity Index scores. $(6.20 \pm 2.77$ vs $5.72 \pm 2.61 ; p=0.009)$. The clinical cure rates and effectiveness for patients receiving cefoperazone-sulbactam and piperacillin-tazobactam were $84.2 \%$ versus $80.3 \%(p=0.367)$ and $85.4 \%$ versus $83.3 \%(p=0.258)$, respectively. In addition, the overall mortality rate of the cefoperazone-sulbactam group was $16 \%(n=55)$, which was also comparable to the piperacillin-tazobactam group $(17.8 \%, n=84, p=0.572)$. The primary clinical outcomes for patients receiving cefoperazone-sulbactam were superior compared to those receiving piperacillin-tazobactam after adjusting disease severity status. The clinical efficacy of cefoperazone-sulbactam in the treatment of adult patients with SCAP is comparable to that of piperacillin-tazobactam. After adjusting for disease severity, cefoperazone-sulbactam tended to be superior to piperacillin-tazobactam.

\section{Introduction}

Community-acquired pneumonia (CAP), and, in particular, severe CAP (SCAP) are significant causes of morbidity and mortality. In spite of evolving and improving therapeutic strategies, ${ }^{1}$ the mortality rate of patients with SCAP remains high, ranging from $17 \%$ to more than $50 \% .{ }^{2,3}$ According to the American Thoracic Society/ Infectious Disease Society of America (ATS/IDSA) guidelines, SCAP is defined as follows: (1) having $\geq 1$ of the major criteria, including invasive mechanical ventilation (IMV) and/or septic shock; (2) having $\geq 3$ of the minor criteria, including: respiratory rate $\geq 30$ breaths/min, partial pressure of oxygen $(\mathrm{PaO} 2) /$ fraction of inspired oxygen (FiO2) $\leq 250 \mathrm{mmHg}$, multiple lobar infiltrates, confusion, blood urea nitrogen (BUN) $\geq 20 \mathrm{mg} / \mathrm{dL}$, white blood cell count $(\mathrm{WBC})<4000$ cells $/ \mathrm{mm} 3$, platelet count $<10,0000 \mathrm{cells} / \mathrm{mm} 3$, temperature $<36^{\circ} \mathrm{C}$, and/or hypotension requiring aggressive fluid resuscitation. ${ }^{4}$ SCAP patients with respiratory failure and IMV, severe sepsis or septic shock, and decompensating comorbidities are at increased risk of death. ${ }^{5,6}$

Streptococcus pneumoniae remains the most common pathogen reported in CAP and is also frequently a cause of SCAP, which generally results in intensive care unit (ICU) admission. ${ }^{7,8}$ Haemophilus influenza, Legionella pneumonia, and influenza pneumonia are pathogens that also result in SCAP in the ICU.9,10 However, about $6 \%$ of pathogens outside the core microorganisms of CAP, called the PES (Pseudomonas aeruginosa, extended-spectrum $\beta$-lactamase Enterobacteriaceae, and methicillin-resistant Staphylococcus aureus), can also cause severe illness ${ }^{11}$, and the rate of inadequate use of empiric antibiotics is very high. It is important to identify the risk factors of PES in order to choose appropriate antibiotic treatment. The main components of the PES score are as follows: age > 65 years old, male gender, previous antibiotic 
usage, presence of chronic respiratory disorder, presence of chronic renal disease, impaired consciousness, and fever. ${ }^{12}$ Marayuma et al. have indicated an antibiotic strategy for pneumonia based on the risk factors for PES pathogens, rather than the site of pneumonia acquisition, as these factors impact the patient's PES score. ${ }^{13}$ The use of empiric broad-spectrum antibiotics that cover PES pathogens should be considered to improve patient outcomes, especially in those with a PES score $\geq 5 .{ }^{14}$

Cefoperazone-sulbactam is a broad-spectrum antibacterial agent and is active against commonly encountered respiratory pathogens and multidrug-resistant organisms (MDROs), including Enterobacteriaceae members, pseudomonas aeruginosa, other non-glucose-fermenting gram-negative bacilli, and anaerobes. ${ }^{15}$ Evidence suggests that cefoperazone-sulbactam is not inferior to cefepime and piperacillin-tazobactam for treating hospital-acquired pneumonia (HAP) and healthcare-associated

pneumonia (HCAP). ${ }^{16,17}$ However, studies regarding the clinical effectiveness of cefoperazone-sulbactam in the management of SCAP are lacking. The current study was conducted to clarify the efficacy and safety of cefoperazone-sulbactam versus piperacillin-tazobactam in the treatment of SCAP.

\section{Results}

\section{The demographic data}

A total of 815 patients with SCAP were enrolled. The baseline characteristics of enrolled patients are shown in Table 1. Among these patients, 343 received cefoperazone-sulbactam, and 472 received piperacillin-tazobactam. The mean age of patients in the cefoperazone-sulbactam group was $74.78 \pm$ 15.00 years, and $242(70.6 \%)$ were male. The median treatment duration of cefoperazone-sulbactam was $9.08 \pm 3.53$ days. Similar baseline characteristics were noted across both groups, including data related to age, gender, and duration of antibiotic treatment; however, the Charlson Comorbidity Index was significantly higher in the cefoperazone-sulbactam group compared to the piperacillin-tazobactam group $(6.20 \pm 2.77$ vs $5.72 \pm 2.61$, respectively; $p=0.009)$. In contrast, there were no significant differences between the two groups in the Pneumonia Severity Index (PSI) scores or the CURB-65 scores. Additionally, the difference of the microbial identification rate between these two groups was not statistically significant $(49.7 \%$ vs $49.6 \%$, respectively; $p=0.981)$. In the cefoperazone-sulbactam group, $K$. pneumonia $(11.1 \%)$ and $P$. aeruginosa $(6.4 \%)$ were the most two commonly identified pathogens. Similar findings were observed in the piperacillin-tazobactam group. The two most common pathogens identified were $P$. aeruginosa (8.6\%) and K. pneumonia (8.2\%). S. pneumoniae was not a commonly identified pathogen in patients with SCAP in the current study (4.8\%). Regarding adverse events, only 21 patients taking cefoperazone-sulbactam had received an international normalized ratio (INR) test pre- or post-therapy after one week. There was no significant difference between the pre-and post-therapy INR in those patients (1.14 vs $1.13 ; p=0.597)$. 
Table 1

Baseline characteristics of patients with SCAP

\begin{tabular}{|c|c|c|c|}
\hline & $\begin{array}{l}\text { cefoperazone-sulbactam } \\
\mathrm{N}=343\end{array}$ & piperacillin-tazobactam N=472 & $p$-value \\
\hline Age (years) Mean \pm SD & $74.78 \pm 15.00$ & $75.14 \pm 14.20$ & 0.874 \\
\hline Gender, Male (\%) & $242(70.6 \%)$ & $339(71.8 \%)$ & 0.696 \\
\hline Treatment days Mean \pm SD & $9.08 \pm 3.53$ & $9.31 \pm 3.75$ & 0.462 \\
\hline \multicolumn{4}{|l|}{ Pathogens, n (\%) } \\
\hline Escherichia coli & $18(5.2 \%)$ & $34(7.2 \%)$ & 0.325 \\
\hline Klebsiella pneumoniae & $38(11.1 \%)$ & $39(8.2 \%)$ & 0.216 \\
\hline Pseudomonas aeruginosa & $22(6.4 \%)$ & $41(8.6 \%)$ & 0.286 \\
\hline Acinetobacter spp. & $20(5.8 \%)$ & $21(4.4 \%)$ & 0.466 \\
\hline Streptococcus & $19(5.5 \%)$ & $20(4.2 \%)$ & 0.487 \\
\hline Other pathogens & $50(14.5 \%)$ & $74(15.6 \%)$ & 0.739 \\
\hline No growth/NA & $176(51.3 \%)$ & $243(51.4 \%)$ & 0.981 \\
\hline Charlson score, Mean \pm SD & $6.20 \pm 2.77$ & $5.72 \pm 2.61$ & $0.009^{*}$ \\
\hline PSI score, Mean \pm SD & $146.89 \pm 45.34$ & $146.63 \pm 43.08$ & 0.651 \\
\hline Curb-65 score, Mean \pm SD & $2.65 \pm 1.23$ & $2.60 \pm 1.30$ & 0.649 \\
\hline In-hospital Mortality, n (\%) & $55(16.0 \%)$ & $84(17.8 \%)$ & 0.572 \\
\hline Primary outcome, n (\%) & & & 0.367 \\
\hline Clinical cure & $287(84.2 \%)$ & $378(80.3 \%)$ & \\
\hline Failure/ Indeterminate & $54(15.7 \%)$ & $93(19.7 \%)$ & \\
\hline Secondary outcome, n (\%) & & & 0.258 \\
\hline Effective & $293(85.4 \%)$ & $393(83.3 \%)$ & \\
\hline Ineffective /Indeterminate & $50(14.5 \%)$ & 79(16.7\%) & \\
\hline INR before medicine, $n$ & 29 & 11 & 0.296 \\
\hline Median (95\% Cl) & $1.14(1.07,1.19)$ & $1.10(0.90,1.82)$ & \\
\hline INR after medicine, $n$ & 21 & 6 & 0.579 \\
\hline Median $(95 \% \mathrm{Cl})$ & $1.13(1.09,1.25)$ & $1.06(0.94,1.33)$ & \\
\hline
\end{tabular}




\section{$\mathrm{N}=343$}

Cl: Confidence interval; INR: international normalized ratio; PSI: Pneumonia Severity Index; SCAP: Severe community acquired pneumonia; SD: Standard deviation. ${ }^{\star} p<0.05$

\section{The clinical characteristics of patients with and without PES}

PES pathogens were identified in $8.7 \%$ (71/815) of patients with SCAP in our cohort (Table 2). Many of the baseline characteristics in both groups were similar, including age, gender, and underlying comorbidities; however, the prevalence of chronic pulmonary disease was higher in the PES group (36.6\% vs $25.8 \%, p=0.049$ ). There were no significant differences in the Charlson Comorbidity Index, APACHE II scores, Sequential Organ Failure Assessment (SOFA) scores, and Quick SOFA (qSOFA) scores when comparing the PES and non-PES groups. In spite of these circumstances, the clinical cure rate of patients in the PES group was significantly lower than the rate for the non-PES group $(73.2 \%$ vs $82.9 \%, p=0.021)$. The in-hospital mortality rate for patients in the PES group was higher than it was for those in in non-PES group $(26.8 \%$ vs $16.1 \%, p=0.023)$. 
Table 2

Baseline characteristics of patients with PES or non/unknown PES group

\begin{tabular}{|c|c|c|c|}
\hline & $\begin{array}{l}\text { PES group } \\
\mathrm{N}=71\end{array}$ & $\begin{array}{l}\text { Non-PES or unknown group } \\
\mathrm{N}=744\end{array}$ & $p$-value \\
\hline Age (years) Mean \pm SD & $72.46 \pm 14.02$ & $75.23 \pm 14.57$ & 0.073 \\
\hline Gender, Male, n (\%) & $51(71.8 \%)$ & $530(71.2 \%)$ & 0.916 \\
\hline Myocardial infarction, n (\%) & $2(2.8 \%)$ & $54(7.3 \%)$ & 0.158 \\
\hline Congestive heart failure, $\mathrm{n}(\%)$ & $8(11.3 \%)$ & $107(14.4 \%)$ & 0.471 \\
\hline Peripheral vascular disease, $n$ (\%) & $2(2.8 \%)$ & $19(2.6 \%)$ & 0.894 \\
\hline CVA, n (\%) & $5(7.0 \%)$ & 77 (10.3\%) & 0.376 \\
\hline Dementia, n (\%) & $12(16.9 \%)$ & $134(18.0 \%)$ & 0.816 \\
\hline Chronic pulmonary disease, $\mathrm{n}(\%)$ & $26(36.6 \%)$ & $192(25.8 \%)$ & $0.049^{*}$ \\
\hline Connective tissue disease, n (\%) & $4(5.6 \%)$ & $38(5.1 \%)$ & 0.848 \\
\hline Peptic ulcer disease, n (\%) & $7(9.9 \%)$ & $70(9.4 \%)$ & 0.901 \\
\hline Liver disease, n (\%) & $3(4.2 \%)$ & $50(6.7 \%)$ & 0.415 \\
\hline Diabetes mellitus, n (\%) & $22(31.0 \%)$ & $263(35.3 \%)$ & 0.461 \\
\hline Hemiplegia, n (\%) & $10(14.1 \%)$ & $101(13.6 \%)$ & 0.905 \\
\hline Moderate to severe CKD, n (\%) & $9(12.7 \%)$ & $124(16.7 \%)$ & 0.385 \\
\hline Solid tumor, n (\%) & $7(9.9 \%)$ & $69(9.3 \%)$ & 0.871 \\
\hline Leukemia, n (\%) & $0(0.0 \%)$ & $7(0.9 \%)$ & 0.412 \\
\hline Lymphoma, n (\%) & $0(0.0 \%)$ & $1(0.1 \%)$ & 0.757 \\
\hline AIDS, n (\%) & $0(0.0 \%)$ & $3(0.4 \%)$ & 0.592 \\
\hline Charlson Score & $5.61 \pm 2.70$ & $5.95 \pm 2.69$ & 0.264 \\
\hline APACHE II Score & $20.63 \pm 8.79$ & $22.15 \pm 7.05$ & 0.250 \\
\hline SOFA Score & $6.24 \pm 3.80$ & $7.02 \pm 3.75$ & 0.344 \\
\hline qSOFA Score & $1.92 \pm 0.51$ & $1.74 \pm 0.74$ & 0.459 \\
\hline Treatment duration (days) & $10.25 \pm 4.84$ & $9.11 \pm 3.51$ & 0.108 \\
\hline Primary outcome, n (\%) & & & $0.021^{*}$ \\
\hline Clinical cure & $51(71.8 \%)$ & $614(82.9 \%)$ & \\
\hline
\end{tabular}




\begin{tabular}{|c|c|c|c|}
\hline & $\begin{array}{l}\text { PES group } \\
\mathrm{N}=71\end{array}$ & $\begin{array}{l}\text { Non-PES or unknown group } \\
\mathrm{N}=744\end{array}$ & $p$-value \\
\hline Failure/Indeterminate & $20(28.2 \%)$ & $127(17.1 \%)$ & \\
\hline Secondary outcome, n (\%) & & & 0.200 \\
\hline Effective & $56(78.9 \%)$ & $630(84.7 \%)$ & \\
\hline Ineffective/Indeterminate & $15(21.1 \%)$ & $114(15.3 \%)$ & \\
\hline In-hospital mortality, n (\%) & $19(26.8 \%)$ & $120(16.1 \%)$ & $0.023^{*}$ \\
\hline
\end{tabular}

\section{Treatment outcomes}

The primary outcomes of clinical cure rate and failure (or indeterminate) rate between the cefoperazonesulbactam and piperacillin-tazobactam groups were $84.2 \%$ vs $80.3 \%$ and $15.7 \%$ vs $19.7 \%$, respectively ( $p$ $=0.367$ ) (Figure 1A). For the secondary outcomes, the clinical effectiveness rate and ineffective (or indeterminate) rates of cefoperazone-sulbactam were $85.4 \%$ and $14.5 \%$, which were similar to rates for the piperacillin-tazobactam group $(83.3 \%$ and $16.7 \% ; p=0.258)$ (Figure 1B). Furthermore, the in-hospital mortality rate was similar between the cefoperazone-sulbactam and piperacillin-tazobactam groups $(16.0 \%$ vs $17.8 \% ; p=0.572)$ (Table 1$)$. On the other hand, based off of the Charlson scores, disease severity was different between the two groups. The propensity score method was performed to adjust for this confounding factor by adjusting the Charlson score, as seen in the supplementary table 1 . The clinical cure rate of cefoperazone-sulbactam was higher than the cure rate with piperacillin-tazobactam (85.4\% vs 79.3\%; $p=0.041$ )[adjusted odds ratio (OR), 1.53; 95\% confidence interval $(\mathrm{Cl}), 1.02-2.31$ ]. No significant difference was found between the two groups in terms of secondary outcomes or in-hospital mortality (Figure 2 and Table 3 ).

Table 3

Outcome analysis of patients receiving cefoperazone-sulbactam versus piperacillin-tazobactam

\begin{tabular}{|c|c|c|c|c|c|c|}
\hline \multirow[b]{2}{*}{$N=324$} & \multicolumn{2}{|c|}{ Primary Outcome } & \multicolumn{2}{|c|}{ Secondary Outcome } & \multicolumn{2}{|c|}{ In-hospital mortality } \\
\hline & $\begin{array}{l}\text { Crude } \\
\text { OR (95\% } \\
\text { CI) }\end{array}$ & $\begin{array}{l}\text { Adjust } \\
\mathrm{OR}^{*}(95 \% \\
\mathrm{Cl})\end{array}$ & $\begin{array}{l}\text { Crude } \\
\text { OR (95\% } \\
\text { Cl) }\end{array}$ & $\begin{array}{l}\text { Adjust } \\
\mathrm{OR}^{*}(95 \% \\
\mathrm{Cl})\end{array}$ & $\begin{array}{l}\text { Crude OR } \\
(95 \% \mathrm{Cl})\end{array}$ & $\begin{array}{l}\text { Adjust } \\
\mathrm{OR}^{*} \\
(95 \% \mathrm{Cl})\end{array}$ \\
\hline \multicolumn{7}{|c|}{ cefoperazone-sulbactam vs } \\
\hline $\begin{array}{l}\text { piperacillin- } \\
\text { tazobactam }\end{array}$ & $\begin{array}{l}1.53 \\
(1.02 \\
2.31)\end{array}$ & $\begin{array}{l}1.53 \\
(1.02 \\
2.31)\end{array}$ & $\begin{array}{l}1.36 \\
(0.89 \\
2.08)\end{array}$ & $\begin{array}{l}1.36 \\
(0.89 \\
2.08)\end{array}$ & $\begin{array}{l}1.34 \\
(0.90 \\
2.01)\end{array}$ & $\begin{array}{l}1.34(0.90 \\
2.01)\end{array}$ \\
\hline
\end{tabular}

*: adjust OR by Wald method; Cl: Confidence interval; OR: Odds Ratio 


\section{Discussion}

The major findings in the current study can be summarized as follows: First, for the treatment of SCAP, the clinical cure rates and effectiveness rates of cefoperazone-sulbactam were similar to those of piperacillin-tazobactam; however, cefoperazone-sulbactam demonstrated superior clinical primary outcomes following adjustment of Charlson scores. The in-hospital mortality was similar between patients receiving either of these two antibiotics. Finally, PES pathogens were more commonly identified in patients with chronic pulmonary disease, and these patients tended to experience poor outcomes.

The hospital mortality of SCAP is still high, ranging from $17 \%$ to over $50 \%{ }^{2,3}$ Because of the emergence of pathogens outside of the core microorganisms of $\mathrm{CAP}_{1}^{12}$ it is crucial to administer adequate broadspectrum antibiotics covering PES pathogens in the early stages of treatment. ${ }^{18}$ In this study, PES pathogens were identified in $8.7 \%$ of SCAP patients, which is consistent with several previous studies, which identified these pathogens in $6-7 \%$ of CAP patients. ${ }^{12,19}$ In the current study, P. aeruginosa (7.7\%) and $K$. pneumoniae $(9.3 \%)$ were the most two commonly identified pathogens resulting in SCAP. These were followed by E. coli (6.3\%), Acinetobacter spp. (5.0\%), and streptococcus pneumoniae (4.7\%). The hospital mortality rates for SCAP patients receiving cefoperazone-sulbactam and piperacillin-tazobactam were $16.0 \%$ and $17.8 \%$, respectively; however, the hospital mortality rate of SCAP patients with PES pathogens was higher than the rate for those with non-PES pathogens $(26.8 \%$ vs $16.1 \%, p=0.023)$. In our cohort, the mortality rate was lower in patients suffering from SCAP compared to previous reports, which suggests that broad-spectrum antibiotics should be used initially. ${ }^{18}$

Risk factors associated with increased chance of death have been identified, including patient indication of prior antibiotic usage, use of proton pump inhibitor medications, need for tube feeding, nonambulatory status, ${ }^{19}$ CAP severity, recent hospitalization, dialysis, and immunosuppression. ${ }^{13}$ Furthermore, male sex, presence of chronic airway disease, need for vasoactive drug or mechanical ventilation, higher $\mathrm{PSI}$, and prior $P$. aeruginosa colonization have been identified as risk factors for $P$. aeruginosa CAP. ${ }^{11}$ Chronic pulmonary disease was the only risk factor identified for the SCAP patients with PES pathogens in our cohort, which is consistent with the previous report. ${ }^{11}$

Cefoperazone is effective not only against gram-positive aerobes but also against a wide variety of gramnegative aerobes, including Enterobacteriaceae members, $P$. aeruginosa, and other non-glucosefermenting Gram-negative bacilli (GNB) in vitro. ${ }^{20}$ However, its minimum inhibitory concentration is influenced by the high inoculum concentration of B-lactamase-producing organisms. ${ }^{20}$ When combined with sulbactam, the bacterial inoculum effect against cefoperazone can be overcome ${ }^{21}$ and can increase the additional coverage of anaerobes. ${ }^{22}$ In addition, sulbactam contains intrinsic antibacterial activity to combat Acinetobacter species. ${ }^{23}$ Jean SS et al. reported the susceptibility rates of E. coli, K. pneumoniae, Citrobacter freundii, Serratia marcescens, Proteus mirabilis, P. aeruginosa, Enterobacter cloacae, and Acinetobacter spp. to cefoperazone-sulbactam were all above $85 \% .{ }^{24}$ For drug resistant organisms, the susceptibility rates to 
cefoperazone-sulbactam have been shown to be 97\% (ESBL- E. coli), 75.8\% (ESBL- K. pneumoniae), $67.6 \%$ (ESBL- K. pneumoniae), and 68\% (carbapenem-resistant A. baumanni). ${ }^{21}$ Piperacillin-tazobactam was the most commonly prescribed antibiotic for suspected drug resistant pathogens, but it has been shown to have adverse outcomes in patients with $E$. coli and K. pneumoniae bacteremia. ${ }^{25}$ The current study demonstrated a superior clinical cure rate with cefoperazone-sulbactam compared to piperacillintazobactam after adjustment severity scores for SCAP. Based on the research indicated above, cefoperazone-sulbactam has been shown to be another alternative antibiotic to utilize in the fight against antibiotic-resistant bacteria.

On the other hand, there are safety considerations to be taken into account when prescribing cefoperazone-sulbactam. The coagulopathy, due to its N-methylthiotetrazole (NMTT) side chain, potentially induces transient hypoprothrombinemia. ${ }^{26}$ This complication is generally rare and is only observed in patients concurrently receiving anticoagulants, in malnourished patients, and in patients who have experienced hemorrhagic events in the recent half-year. ${ }^{27}$ In the current study, no significant difference in the pre-and post- INR in was observed with the 21 patients receiving cefoperazone; however, most of the patients were not routinely assessed for bleeding disorders because they did not present with significant bleeding in our retrospective study.

We acknowledge some limitations to this study. First, the retrospective design inherently cannot ensure baseline equality between the two groups. The propensity score matching method was performed to adjust the baseline clinical characteristics. Secondly, the PES score was not reported for each patient; however, the severity of CAP was high enough to prescribe broad-spectrum antibiotics. The identification of PES pathogens may have been underestimated, as the microbial identification rate between the two groups was around $50 \%$. Lastly, mild adverse events were not recorded, and some patients did not receive INR measurement. Therefore, the risk of adverse events and the safety of cefoperazone-sulbactam could have been underestimated.

In summary, this study suggests cefoperazone-sulbactam is as effective as piperacillin-tazobactam in the treatment of patients with SCAP. After adjusting for disease severity, the clinical cure rate of cefoperazone-sulbactam tended to be superior to piperacillin-tazobactam in treating SCAP.

\section{Materials And Methods}

\section{Study Design and Data Collection}

This study serves as a multicenter retrospective analysis of the clinical effectiveness and safety of cefoperazone-sulbactam in the management of patients suffering from SCAP. The clinical data were extracted from the BATTLE study, which investigated the efficacy and safety of Brosym(TTY Biopharm Company, Taiwan) in the management of SCAP and nosocomial pneumonia. The study was conducted between March 2018 and May 2019 at eight medical centers and one regional hospital in Taiwan. The subjects enrolled in the study received either cefoperazone-sulbactam or piperacillin-tazobactam in the 
treatment of SCAP, hospital-acquired pneumonia (HAP), and ventilator-associated pneumonia (VAP). The data were collected retrospectively from electronic medical records after obtaining approval from local ethics committees or institutional review boards. Data included the baseline characteristics of patients, their underlying diseases, type of bacteria observed, type of antibiotic treatment utilized, and clinical outcomes. Due to the retrospective design, individual patient consent was not indicated. All methods were performed in accordance with the Declaration of Helsinki.

\section{Definitions and Outcomes}

For the purposes of this study, pneumonia was defined as having two or more respiratory symptoms and signs (including cough, fever, hypothermia, purulent sputum, or respiratory secretions) and opacity on a chest radiograph as interpreted by the attending physician. ${ }^{28}$ The diagnosis of SCAP was based on the ATS and IDSA guidelines. ${ }^{4}$ Both respiratory specimens and blood cultures were sampled prior to the prescription of antibiotics. The primary efficacy endpoint was the clinical cure rate of SCAP in response to the antibiotic treatment. Therefore, the cure rate was defined as the proportion of patients in which the clinical symptoms or signs resolved or improved seven days after the end of antibiotic treatment without additional management. In contrast, clinical failure was defined as follows: clinical symptoms or signs deteriorated or persisted after three to five days of antibiotic treatment and required additional antibiotics for management; death due to pneumonia after three days of antibiotic treatment; or progression of pneumonia and development of empyema or lung abscess. The indeterminate outcomes included transfers to other hospitals, refusal of further treatment, death due to pneumonia following fewer than two days of antibiotic treatment, incomplete antibiotic treatment due to allergy, severe adverse events, or other personal reasons. The secondary outcomes measured included the clinical effective rate, the risk of adverse effects, and in-hospital mortality. The clinical effectiveness was defined as the improvement of clinical symptoms and signs, radiographic opacity, and inflammation profiles, including white blood cell counts, procalcitonin, c-reactive protein levels. In contrast, the clinical ineffectiveness was defined as when any one of the three criteria mentioned above was not achieved. The indeterminate effectiveness was defined as when the above three criteria could not be assessed.

\section{Statistical Analysis}

Data were compiled and analyzed using the Statistical Analysis System(SAS) for Windows (Version 9.4 or higher, SAS Institute, Cary, North Carolina, USA). All continuous variables were reported as means with standard deviation or medians with interquartile range (IQR). Differences in continuous variables were compared using independent t-tests or Kruskal-Wallis tests. Meanwhile, categorical variables were reported as the number of patients and percentages. Differences in categorical variables were examined using the F-test or chi-square test. The difference in the crude relative risk and adjusted relative risk (adjusted for propensity score) of the outcomes between cefoperazone-sulbactam versus piperacillintazobactam were calculated. All tests of significance were two-tailed, and a p-value of $\leq 0.05$ was considered to be statistically significant. 


\section{Abbreviations}

CAP: Community-acquired pneumonia; Cl: confidence interval; GNB: Gram-negative bacilli; HAP: Hospitalacquired pneumonia; INR: international normalized ratio, MDRO: multidrug resistant organism, MIC: minimum inhibitory concentration, NMTT: N-methylthiotetrazole, OR: odds ratio, PSI: Pneumonia severity index; SCAP: Severe community acquired pneumonia; VAP: Ventilator-associated pneumonia

\section{Declarations}

\section{Acknowledgments}

Not applicable.

\section{Funding}

This study was financially supported by the grant from the China Medical University Hospital (Grant number: DMR-107-022).

\section{Availability of data and materials}

Not applicable.

\section{Competing interests}

No conflicts exist for the specified authors.

\section{Consent for publication}

All authors have reviewed and approved the manuscript for publication.

\section{Authors' Contributions}

(I) Conception and design: C.C Lai, C.H Chen, C.Y Tu, W.C Cheng

(II) Administrative support: C.C Lai, C.H Chen, C.Y Tu, W.C Cheng

(III) Provision of study materials or patients: C.C Lai, C.H Chen, C.Y Tu, W.C Cheng, W.C Chen, L.K Kuo, Y-T Wang, P-K Fu, S.C Ku, W.F Fang, C.M Chen

(IV) Collection and assembly of data: C.C Lai, C.H Chen, W.C Cheng 
(V) Data analysis and interpretation: C.C Lai, C.H Chen, W.C Cheng

(VI) Manuscript writing: C.C Lai, C.H Chen, W.C Cheng

(VII) Final approval of manuscript: C.C Lai, C.H Chen, C.Y Tu, W.C Cheng, W.C Chen, L.K Kuo, Y-T Wang, PK Fu, S.C Ku, W.F Fang, C.M Chen

\section{Clinical trial registry number}

Not applicable

\section{Ethical Approval and Consent to Participate}

This study was approved by the ethics committee of Taipei Veterans General Hospital (2019-08-006AC), Mackay Memorial Hospital (19MMHIS282e), Chung Shan Medical University Hospital (CS18126), Taichung Veterans General Hospital (CE18223A\#2), National Taiwan University Hospital (201805123RINB), Kaohsiung Chang Gung Memorial Hospital (201900932B0C501), Chi Mei Medical Center (10806-008) and Chi Mei Medical Center, Liouying (10808-L01).

\section{References}

1. Leoni D, Rello J. Severe community-acquired pneumonia: optimal management. Curr Opin Infect Dis. 2017;30(2):240-247.

2. Phua J, Dean NC, Guo Q, Kuan WS, Lim HF, Lim TK. Severe community-acquired pneumonia: timely management measures in the first 24 hours. Crit Care. 2016;20:237.

3. Montull B, Menendez R, Torres A, et al. Predictors of Severe Sepsis among Patients Hospitalized for Community-Acquired Pneumonia. PLoS One. 2016;11(1):e0145929.

4. Mandell LA, Wunderink RG, Anzueto A, et al. Infectious Diseases Society of America/American Thoracic Society consensus guidelines on the management of community-acquired pneumonia in adults. Clin Infect Dis. 2007;44 Suppl 2:S27-72.

5. Ferrer M, Travierso C, Cilloniz C, et al. Severe community-acquired pneumonia: Characteristics and prognostic factors in ventilated and non-ventilated patients. PLoS One. 2018;13(1):e0191721.

6. Cilloniz C, Ferrer M, Liapikou A, et al. Acute respiratory distress syndrome in mechanically ventilated patients with community-acquired pneumonia. Eur Respir J. 2018;51(3).

7. Walden AP, Clarke GM, McKechnie S, et al. Patients with community acquired pneumonia admitted to European intensive care units: an epidemiological survey of the GenOSept cohort. Crit Care. 2014;18(2):R58.

8. Valles J, Diaz E, Martin-Loeches I, et al. Evolution over a 15-year period of the clinical characteristics and outcomes of critically ill patients with severe community-acquired pneumonia. Med Intensiva. 
2016;40(4):238-245.

9. Shorr AF, Bodi M, Rodriguez A, et al. Impact of antibiotic guideline compliance on duration of mechanical ventilation in critically ill patients with community-acquired pneumonia. Chest. 2006;130(1):93-100.

10. Wiemken T, Peyrani P, Bryant K, et al. Incidence of respiratory viruses in patients with communityacquired pneumonia admitted to the intensive care unit: results from the Severe Influenza Pneumonia Surveillance (SIPS) project. Eur J Clin Microbiol Infect Dis. 2013;32(5):705-710.

11. Cilloniz C, Gabarrus A, Ferrer M, et al. Community-Acquired Pneumonia Due to Multidrug- and NonMultidrug-Resistant Pseudomonas aeruginosa. Chest. 2016;150(2):415-425.

12. Prina E, Ranzani OT, Polverino E, et al. Risk factors associated with potentially antibiotic-resistant pathogens in community-acquired pneumonia. Ann Am Thorac Soc. 2015;12(2):153-160.

13. Maruyama T, Fujisawa T, Ishida T, et al. A Therapeutic Strategy for All Pneumonia Patients: A 3-Year Prospective Multicenter Cohort Study Using Risk Factors for Multidrug-resistant Pathogens to Select Initial Empiric Therapy. Clin Infect Dis. 2019;68(7):1080-1088.

14. Cilloniz C, Dominedo C, Nicolini A, Torres A. PES Pathogens in Severe Community-Acquired Pneumonia. Microorganisms. 2019;7(2).

15. Sader HS, Carvalhaes CG, Streit JM, Castanheira M, Flamm RK. Antimicrobial activity of cefoperazone-sulbactam tested against Gram-Negative organisms from Europe, Asia-Pacific, and Latin America. Int J Infect Dis. 2020;91:32-37.

16. Chen $\mathrm{CH}$, Tu CY, Chen WC, et al. Clinical Efficacy of Cefoperazone-Sulbactam versus PiperacillinTazobactam in the Treatment of Hospital-Acquired Pneumonia and Ventilator-Associated Pneumonia. Infect Drug Resist. 2021;14:2251-2258.

17. Liu JW, Chen YH, Lee WS, et al. Randomized Noninferiority Trial of Cefoperazone-Sulbactam versus Cefepime in the Treatment of Hospital-Acquired and Healthcare-Associated Pneumonia. Antimicrob Agents Chemother. 2019;63(8).

18. Garnacho-Montero J, Barrero-Garcia I, Gomez-Prieto MG, Martin-Loeches I. Severe communityacquired pneumonia: current management and future therapeutic alternatives. Expert Rev Anti Infect Ther. 2018;16(9):667-677.

19. Shindo $Y$, Ito R, Kobayashi $D$, et al. Risk factors for drug-resistant pathogens in community-acquired and healthcare-associated pneumonia. Am J Respir Crit Care Med. 2013;188(8):985-995.

20. Jones RN, Barry AL. Cefoperazone: a review of its antimicrobial spectrum, beta-lactamase stability, enzyme inhibition, and other in vitro characteristics. Rev Infect Dis. 1983;5 Suppl 1:S108-126.

21. Chang PC, Chen CC, Lu YC, et al. The impact of inoculum size on the activity of cefoperazonesulbactam against multidrug resistant organisms. J Microbiol Immunol Infect. 2018;51(2):207-213.

22. Moody JA, Fasching CE, Sinn LM, Gerding DN, Peterson LR. Comparative efficacy of cefoperazone, cefoperazone plus sulbactam, ciprofloxacin, clindamycin, metronidazole, and penicillin $\mathrm{G}$ against anaerobic bacteria in an animal model. J Lab Clin Med. 1990;115(2):190-195. 
23. Ye JJ, Lin HS, Yeh CF, et al. Tigecycline-based versus sulbactam-based treatment for pneumonia involving multidrug-resistant Acinetobacter calcoaceticus-Acinetobacter baumannii complex. BMC Infect Dis. 2016;16:374.

24. Jean SS, Liao CH, Sheng WH, Lee WS, Hsueh PR. Comparison of commonly used antimicrobial susceptibility testing methods for evaluating susceptibilities of clinical isolates of Enterobacteriaceae and nonfermentative Gram-negative bacilli to cefoperazone-sulbactam. $J$ Microbiol Immunol Infect. 2017;50(4):454-463.

25. Harris PNA, Tambyah PA, Lye DC, et al. Effect of Piperacillin-Tazobactam vs Meropenem on 30-Day Mortality for Patients With E coli or Klebsiella pneumoniae Bloodstream Infection and Ceftriaxone Resistance: A Randomized Clinical Trial. JAMA. 2018;320(10):984-994.

26. Wang W, Liu Y, Yu C, et al. Cefoperazone-sulbactam and risk of coagulation disorders or bleeding: a retrospective cohort study. Expert Opin Drug Saf. 2020;19(3):339-347.

27. Chen LJ, Hsiao FY, Shen LJ, et al. Use of Hypoprothrombinemia-Inducing Cephalosporins and the Risk of Hemorrhagic Events: A Nationwide Nested Case-Control Study. PLoS One. 2016;11(7):e0158407.

28. Chou CC, Shen CF, Chen SJ, et al. Recommendations and guidelines for the treatment of pneumonia in Taiwan. J Microbiol Immunol Infect. 2019;52(1):172-199.

\section{Figures}
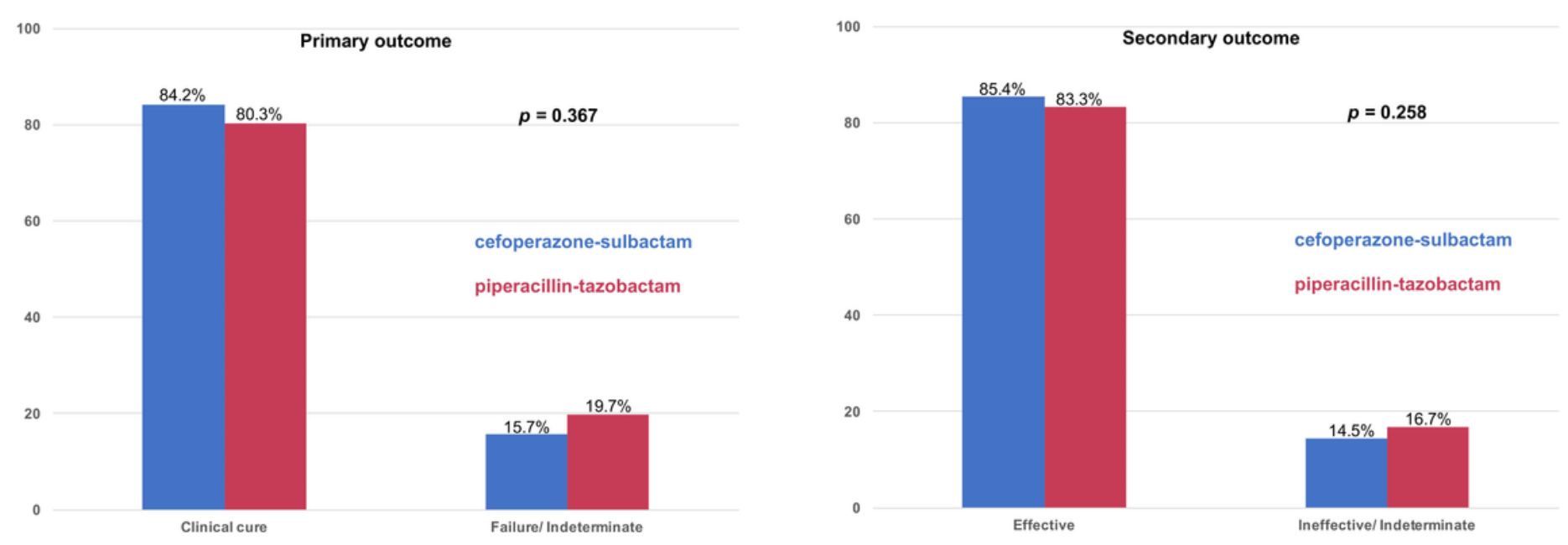

Figure 1

(A) Primary outcome and (B) secondary outcome of patients receiving cefoperazone-sulbactam and piperacillin-tazobactam for severe community - acquired pneumonia 


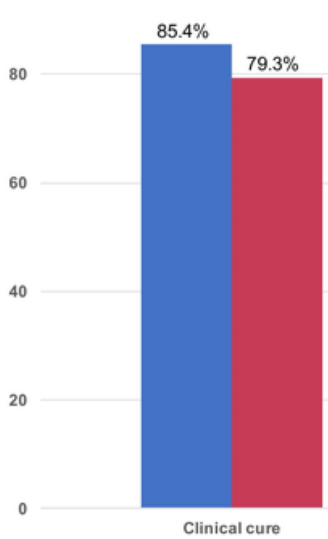

Primary outcome

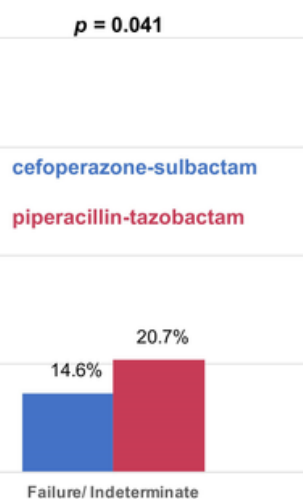

100

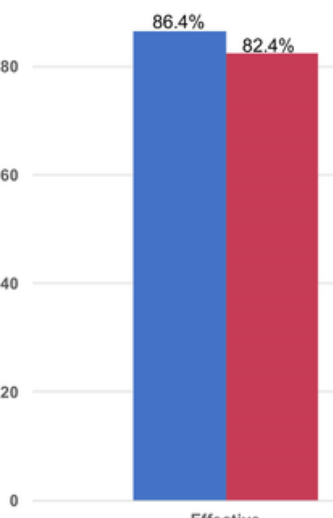

Effective
Secondary outcome

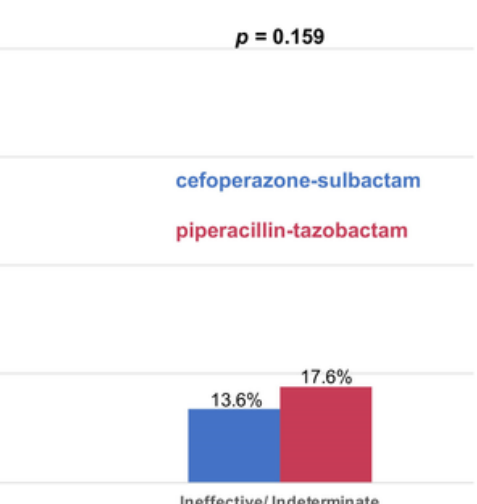

Figure 2

(A) Primary outcome and (B) secondary outcome of patients receiving cefoperazone-sulbactam and piperacillin-tazobactam for severe community - acquired pneumonia after adjusting propensity score. 